\title{
La educación formal de los formadores de la era digital - los educadores del siglo XXI
}

\author{
Julio Cabero Almenara ${ }^{1}$ \\ Universidad de Sevilla \\ Verónica Marín Díaz ${ }^{2}$ \\ Universidad de Córdoba
}

\begin{abstract}
Resumen: La sociedad del conocimiento se presenta como un nuevo escenario, con características distintivas frente a la sociedad industrial y postindustrial, siendo una de sus elementos clave la alta penetración de las Tecnologías de la Información y Comunicación en todos sus sectores, desde el industrial hasta el educativo. En este los docentes se encuentren con nuevos escenario virtuales en los cuales deben de llevar a cabo el proceso de instrucción. Pero esta transformación en las instituciones educativas no se ha limitado a la incorporación de las TIC, sino también a la introducción de un nuevo tipo de alumnos, que al nacer en una sociedad digital y al interaccionar con las tecnologías, presenta características cognitivas diferentes a los alumnos del siglo anterior. En estas líneas se hace un análisis del nuevo escenario en aue los educadores se mueven.

Palabras clave: TIC, formación del profesorado, nuevos entornos de formación, sociedad del conocimiento, roles del docente.

Abstract: The knowledge society is presented as a new scene, with distinctive characteristics front to industrial and post-industrial society, one of its key high penetration Information and communication technologies in all areas elements, from industrial to educational. In this teachers are with new virtual scene which must bring about the training process. But this transformation in educational institutions has not limited to incorporation the ICT, but also the introduction of new type of students, who born in a digital society and interact with technologies, presents cognitive characteristics different from students of previous century. In these lines an analysis of the new scene in which educators move is made.
\end{abstract}

Keywords: ICT, training teacher, new training environments, knowledge society, teachers roles.

\section{1.- Cambios en la sociedad de la información}

Todos estaremos de acuerdo con que en los últimos tiempos se han producido notables cambios como consecuencia de diferentes transformaciones, la más significativa de ellas, el pasar de un modelo de sociedad industrial o postindustrial a otra de la información o el conocimiento, que como señalan Cabero y Barroso (2013) viene marcada a grandes rasgos por las siguientes características: tendencia hacia la

\footnotetext{
${ }^{1}$ Catedrático de Tecnología educativa de la Universidad de Sevilla, Director del Secretariado de Recursos Audiovisuales y Nuevas Tecnologías de la US, Editor Jefe de la revista científica Pixel Bit, Revista de Medios y Educación. Miembro del Claustro de la Universidad de Sevilla. Su línea docente e investigadora se relaciona con la tecnología educativa así como a su uso y aplicabilidad para la mejora de los contextos educativos, las cuales se observan en las diferentes publicaciones, aportaciones a congresos y participación en proyectos de investigación e innovación. Es evaluador de diferentes revistas indexadas nacionales e internacionales.

${ }^{2}$ Profesora Titular de Educación Mediática de Universidad de la Universidad de Córdoba. Editora jefe de la revista digital científica EDMETIC, Revista de Educación Mediática y TIC. Ha sido directora del máster en Educación Inclusiva de la Universidad de Córdoba de 2011 a 2015. Miembro del grupo Comunicar, del que fue Coordinadora provincial de 2004 a 2014. Miembro del Claustro de la Universidad de Córdoba. Su línea docente e investigadora se relaciona con la tecnología educativa así como a su uso y aplicabilidad para la mejora de los contextos educativos, las cuales se observan en las diferentes publicaciones, aportaciones a congresos y participación en proyectos de investigación e innovación nacionales e internacionales. Es evaluadora de diferentes revistas indexadas nacionales e internacionales.
} 
globalización, girar en torno a las Tecnologías de la Información y Comunicación (TIC), transformación de los conceptos de espacio y tiempo, la amplitud y rapidez con la que se genera la información y es puesta disposición de los usuarios, es una sociedad de aprender a aprender, la incorporación de las TIC no es uniforme en todos los sectores, es una sociedad con un fuerte dinamismo y ser una sociedad de redes.

Tales características han transformado la educación y los procesos de aprendizaje en una serie de direcciones entre las cuales podemos destacar: 1) Transformación y velocidad de cambio; 2) Las instancias educativas regladas dejarán de ser las únicas instancias de formación; 3) Transformación de las concepciones del aprendizaje. Aprendizaje colaborativo y distribuido; 4) Entornos altamente tecnificados; 5) La articulación del aprendizaje en torno a lo sincrónico y asincrónico; 6) Formación del estudiante en nuevas competencias y capacidades; 7) Cambios en las estructuras organizativas; 8) La necesidad de configurar redes de formación; 9) Movilidad virtual del estudiante; y 10) Nuevos roles del profesor. Dentro de estos cambios dos de los más significativos, para el objeto que vamos a tratar en la presente intervención, se refieren, por una parte a las transformaciones de las concepciones del aprendizaje, y por otra a la aparición de en los centros educativo de una verdadera de TIC puestas a disposición de los docentes y discentes.

En lo que se refiere al primero, nos encontramos que diversas y nuevas son las teorías psicológicas que están fundamentando en cómo se adquiere el aprendizaje en la sociedad del conocimiento a diferencia de cómo se adquiría en la sociedad postindustrial, el cual venía marcado por la estabilidad y durabilidad de los contenidos, así como por un volumen que permitía que con la memoria humana se pudieran manejar. Como señala Prensky (2011, p.111)

"una de las grandes diferencias entre enseñar en el siglo XXI y en el pasado es que en el pasado las cosas no cambiaban muy deprisa. Así que los profesores preparaban a sus alumnos para un mundo que era muy parecido a aquel en el que estaban viviendo. Pero esa situación ha cambiado ahora de forma drástica. El mundo en que nuestros alumnos vivirán y trabajarán será radicalmente distinto a aquel en el que ellos y nosotros estamos viviendo ahora. Hay que respetar el pasado, por supuesto, pero nuestros alumnos no vivirán en él".

Entre estas nuevas teorías nos encontramos con el conectivismo, el aprendizaje ubicuo, el aprendizaje rizomático y el aprendizaje autorregulado (Cabero y Llorente, 2015). Tales transformaciones nos llevan a pensar que:

“- Cada vez se acepta con mayor facilidad que el aprendizaje es un proceso activo y no pasivo en el que la participación del estudiante es clave en el proceso.

- Se asume que el gran desafío al que se enfrenta el estudiante, y por tanto los profesores, es que debe producir conocimiento y no simplemente reproducirlo.

- Se admite que el aprendizaje es un proceso social en el que el sujeto, en la interacción con sus compañeros y con el resto de variables curriculares, modifica su estructura cognitiva.

- En el aprendizaje están implicados factores cognitivos y metacognitivos, motivacionales y emotivos y sociales y culturales. 
- La importancia del aprendizaje colaborativo.

- Es integrado, contextualizado y situado, en función de los problemas, necesidades y ubicación espacial del sujeto.

- Deberemos movilizar diferentes sistemas simbólicos para desarrollar e impulsar los distintos tipos de inteligencias.

- La educación cada vez será más personalizada, de modo que se respeten los ritmos, los estilos de aprendizajes y las inteligencias múltiples de cada uno de los alumnos.

- La evaluación no debe referirse únicamente a los productos, sino a los procesos que los han generado, y no debe limitarse a uno de los actores, alumnos, del proceso" (Cabero y Barroso, 2013, p.27).

Otra de las transformaciones la encontramos en el volumen de TIC a las que los docentes y discentes tienen acceso en la sociedad del conocimiento. $\mathrm{Y}$ a esta fuerte presencia se les irán progresivamente incorporando otras, como están poniendo de manifiesto diferentes informes Horizonte (Durall, Gros, Maina, Johnson y Adams, 2012; Johnson, Adams, Cummins, Estrada, Freeman y Hall, 2016) o los reporte EduTrends del Instituto Tecnológico de Monterrey (2015), -Web semántica, Internet de las cosas, analíticas de aprendizaje, realidad aumentada, computación en nubes, MOOC, gamificación, entornos personales de aprendizaje, redes sociales,...-. Todo ello permitirá al docente contar con un verdadero ecosistema digital que nos debe llevar a replantearnos algunas de las ideas que se han empleado sobre la incorporación de las TIC a los contextos de formación.

Retomando el tema de la amplitud tecnológica, es cierto que algunos pueden pensar que algunas de estas tecnologías son futuribles, y lo es, pero no debemos de olvidarnos que el futuro ya está aquí, lo que pasa es que no está bien distribuido. El problema estriba en que la innovación no llega a todos al mismo tiempo, y con ello surge una nueva marginalidad y exclusión social (Cabero, 2015a).

De todas formas no debemos olvidarnos, que muchas veces mejor que pensar en tecnologías futuras, lo que debemos hacer es invertir esfuerzos en construir modelos de enseñanza para obtener el máximo partido de las tecnologías que tenemos actualmente en nuestros centros educativos. La innovación no se consigue por la novedad de aplicación tecnológica, sino por la aplicación de criterios para conseguir nuevos escenarios formativos y comunicativos.

Y ante esta situación bien estará realizar algunas reflexiones respecto a cómo deben ser incorporadas, más aún cuando ha aparecido una, como es Internet, que de verdad está transformando e impactando al sistema educativo, sus funciones, los roles que desempeñan los que participan en el mismos y los lugares donde se alcanza el conocimiento. Como en otro momento histórico lo hizo otro medio, el libro de texto.

Y ello, se debe fundamentalmente a que su incorporación en los procesos de enseñanza-aprendizaje ha sido más como elementos aislados e independientes que como integrados en el currículum y con los elementos que lo conforman (objetivos, metodologías, aspectos organizativos, contenidos, características de los estudiantes,...) (Cabero, 2015a; Cabero y Barroso, 2013). Se puede decir, que muchas veces lo que se ha hecho con su incorporación es buscar cambios en la coreografía externa, cuando en realidad lo que se debería buscar son cambios en la interna, y los modelos de incorporación son muy extensos (Badia, Meneses y García, 2015). 
Todo ello nos está llevando a una transformación del aprendizaje hacia lo que se denominan como e-learning 2.0, que supera la simple unificación de clásicas tecnologías utilizadas en la formación virtual con las herramientas surgidas de la Web 2.0, e implica un cambio en la concepción del alumno como consumidor a proconsumidor, de la recepción pasiva de la información por parte del estudiante a su participación en la construcción del conocimiento y la interactividad, de pasar de productos a procesos centrados en el desempeño y a la adquisición de competencias, de la evaluación sumativa a la auténtica, y del intercambio en clase al intercambio en la comunidad.

\section{2.- Un nuevo tipo de alumno}

No se debe olvidar que también han cambiado los alumnos que se acercan a los centros educativos, que no presentan las mismas características, ni las mismas formas de analizar y enfrentarse a la complejidad del mundo, ni las mismas formas de relacionarse y comunicarse con sus compañeros.

"La aparición de este nuevo tipo de alumnos, como consecuencia, principalmente, del entramado sociocultural y tecnológico en el que se desenvuelven a partir de su momento histórico de nacimiento, ha llevado a que algunos autores propongan términos específicos para hacer referencias a ellos, conceptos que se están desenvolviendo con verdadera naturalidad en nuestra cultura como los de: nativos digitales, generación red, generación mouse, generación Google o generación Einstein; aludiendo con ello a la importancia que las tecnologías, y fundamentalmente la red, tienen en su vida y en las acciones que realizan... lo que no podemos perder de vista es que la apropiación cultural e ideológica de una tecnología no sólo repercute en cómo codificamos y transmitimos la información, sino también, y creo que es lo verdaderamente importante, en los procesos cognitivos que movilizamos y en las direcciones en las que lo hacemos para codificar y decodificarla, y es precisamente en estos procesos donde se modifica nuestra estructura cognitiva para el procesamiento de la información" (Cabero, 2016, p.205)

Tales transformaciones nos las encontramos en que poseen un procesamiento fragmentado, discontinuo e hipermedia, donde adquieren la información de diferentes medios y recursos, con diferentes sistemas simbólicos, y la mezcla y la remezcla de la información; el poseer una capacidad multitarea, y por otra que lo

"Lo amplio y monotemático llega a aburrirles, prefieren lo diverso, flexible y el cambio constante de actividad. Este último comentario pudiera explicar alguno de los realizados por los profesores, respecto a la falta de concentración y a los problemas de motivación que tienen sus alumnos. Prefieren, por ejemplo, aprender el funcionamiento de un programa informático por ensayo y error que leer su manual de funcionamiento, o valoran la información resumida más que los informes fuertemente detallados" (Cabero, 2016, p.207).

Thompson (2013), realiza un trabajo donde analiza específicamente las características cognitivas que presentan los nativos digitales, y los beneficios y riesgos que las mismas le conllevan, en la tabla 1 se presentan las aportaciones que realiza el autor anteriormente citado. 
Tabla 1. Características de los nativos digitales. Fuente: Thompson (2013).

\begin{tabular}{|c|c|c|}
\hline Características & Beneficios potenciales & Riesgos potenciales \\
\hline $\begin{array}{l}\text { Deseo de la velocidad y la } \\
\text { incapacidad para tolerar el } \\
\text { ritmo lento en el medio } \\
\text { ambiente }\end{array}$ & $\begin{array}{l}\text { Capacidad para analizar el texto } \\
\text { y los procesos de información } \\
\text { de forma rápida. }\end{array}$ & $\begin{array}{l}\text { Interfiere en la lectura profunda y } \\
\text { en la reflexión, y en el desarrollo } \\
\text { del pensamiento abstracto }\end{array}$ \\
\hline $\begin{array}{l}\text { Deseo o necesidad percibida } \\
\text { de realizar múltiples tareas }\end{array}$ & $\begin{array}{l}\text { Evita el aburrimiento; los } \\
\text { nativos digitales pueden regular } \\
\text { la multitarea cuando sea } \\
\text { necesario. }\end{array}$ & $\begin{array}{l}\text { Interfiere con la memoria; causa } \\
\text { tiempo de estudio ineficiente; } \\
\text { está asociado con graduaciones } \\
\text { inferiores.; causa agotamiento } \\
\text { mental. }\end{array}$ \\
\hline $\begin{array}{l}\text { Preferencias por las imágenes } \\
\text { en vez del texto }\end{array}$ & $\begin{array}{l}\text { Pueden desarrollar habilidades } \\
\text { visuales especiales }\end{array}$ & $\begin{array}{l}\text { Las habilidades visuales pueden } \\
\text { adquirirse a la expensa de la } \\
\text { lectura profunda, y la habilidad } \\
\text { reflexiva. }\end{array}$ \\
\hline $\begin{array}{l}\text { Tendencia a procesar la } \\
\text { información de manera no } \\
\text { lineal }\end{array}$ & $\begin{array}{l}\text { Múltiples perspectivas } \\
\text { simultáneas aumentan el } \\
\text { potencial para una mayor } \\
\text { comprensión del dominio de } \\
\text { contexto no estructurados de la } \\
\text { complejidad inherente }\end{array}$ & $\begin{array}{l}\text { Pérdida de la capacidad para leer } \\
\text { de manera lineal incluso cuando } \\
\text { le lectura lineal es más } \\
\text { productiva. }\end{array}$ \\
\hline $\begin{array}{l}\text { Preferencia por la colaboración } \\
\text { y la conectividad constante }\end{array}$ & $\begin{array}{l}\text { Uso personal de las redes en } \\
\text { líneas para mejorar el } \\
\text { aprendizaje; contribución a } \\
\text { proyectos auténticos como } \\
\text { Wikipedia que pueden hacer el } \\
\text { aprendizaje relevante }\end{array}$ & $\begin{array}{l}\text { vo en el enfoque de } \\
\text { ón en el aprendizaje }\end{array}$ \\
\hline $\begin{array}{l}\text { Preferencia por el aprendizaje } \\
\text { a través de la actividad en } \\
\text { lugar de leer y escuchar. }\end{array}$ & $\begin{array}{ll}\text { Capacidad para resolver las } \\
\text { cosas antes de recibir } \\
\text { instrucción }\end{array}$ & $\begin{array}{l}\text { La impaciencia pro la instrucción } \\
\text { guiada puede impedir el } \\
\text { aprendizaje del contenido } \\
\text { esencial }\end{array}$ \\
\hline $\begin{array}{l}\text { Mezcla del trabajo y juego; } \\
\text { uso del tiempo libre }\end{array}$ & $\begin{array}{l}\text { Una cognición lúdica - una } \\
\text { tendencia a la prueba de } \\
\text { hipótesis imaginativa - puede } \\
\text { mejorar el aprendizaje. }\end{array}$ & $\begin{array}{l}\text { La expectativa de que le profesor } \\
\text { debe entretener puede mejorar el } \\
\text { aprendizaje autorregulado }\end{array}$ \\
\hline $\begin{array}{l}\text { Expectativas para la } \\
\text { retroalimentación } \\
\text { recompensa inmediata como se } \\
\text { encuentra en los juegos. }\end{array}$ & $\begin{array}{l}\text { Podría aumentar la motivación } \\
\text { en entornos de aprendizaje } \\
\text { donde la retroalimentación } \\
\text { inmediata está presente }\end{array}$ & $\begin{array}{l}\text { La incapacidad para preservar el } \\
\text { rostro del aburrimiento, a corto } \\
\text { plazo podría menoscabar (o pon } \\
\text { afectar) el aprendizaje }\end{array}$ \\
\hline $\begin{array}{l}\text { La preferencia por los } \\
\text { contextos de fantasía que se } \\
\text { encuentran en los juegos y en } \\
\text { la TV realistas y en las } \\
\text { películas. }\end{array}$ & $\begin{array}{l}\text { Aumento potencial de la } \\
\text { motivación y } \\
\text { contextualización } \\
\text { aprendizaje en ambientes de } \\
\text { aprendizaje donde los contextos } \\
\text { de fantasía están presente. }\end{array}$ & $\begin{array}{l}\text { La dependencia de la fantasía o } \\
\text { la narrativa puede ser } \\
\text { improductiva, ya que no es } \\
\text { práctica para todos los tipos de } \\
\text { aprendizaje que no son diseñados } \\
\text { de esa manera. }\end{array}$ \\
\hline $\begin{array}{l}\text { Expectativa de que la } \\
\text { tecnología es parte del paisaje; } \\
\text { dificultad con contextos que } \\
\text { carecen de tecnología }\end{array}$ & $\begin{array}{l}\text { El buen uso pedagógico de la } \\
\text { tecnología puede mejorar la } \\
\text { comprensión conceptual }\end{array}$ & $\begin{array}{l}\text { La novedad de la tecnología } \\
\text { puede distraer; los estudiantes } \\
\text { con menos competencia } \\
\text { tecnológica pueden tener peso } \\
\text { cognitivo superfluo. }\end{array}$ \\
\hline
\end{tabular}

En esta línea cuando Prensky (2011, p.13) les pregunta a los alumnos en un estudio sobre cómo desea que sus profesores le den las clases y realicen la acción docente, encuentra respuestas como las siguientes: no quieren charlas teóricas, quieren seguir sus pasiones e intereses, quieren crear usando las herramientas de su tiempo, quieren trabajar con sus compañeros en grupos de trabajo y proyectos, quieren tomar decisiones y compartir el control, quieren conectar con sus iguales para expresar y compartir sus opiniones, quieren cooperar y competir entre sí, y quieren una educación que no sea únicamente relevante sino también conectada con la realidad. 
Pero una de las transformaciones más significativas, y que está ocupando una cierta mitología, hace referencia a lo altamente competente que son los jóvenes, de forma que se les tiende a platear como "nativos digitales", por haber nacido en un período altamente tecnificado, mientras por el contrario a los docentes se les considera como "inmigrantes digitales" por su momento temporal de nacimiento.

Este hecho, se hace más necesario, si tenemos en cuenta como desde diferentes investigaciones se están poniendo de manifiesto que las competencias digitales de los estudiantes no son tan amplias como cabría esperar, por los razonamientos que desde ciertos sectores y colectivos se realizan al argumentar que los adolescentes y jóvenes por ser "nativos digitales", llevan integrados la capacitación tecnológica, cuando muchas veces ocurre lo contrario (Castaño, Duart y Sancho, 2012; Flores y Del Arco, 2013; Pons, 2013; Romero y Minelli, 2011). Sin olvidarnos, como ponen de manifiesto Ryberg, Dirckink-Holmfeld y Jones (2011), que el colectivo de los jóvenes, no es un homogéneo en lo referido a la competencia digital. Por ello algunos, autores apuntan que mejor que denominarlos nativos digitales, sería más correcto referirnos a ellos como estudiantes digitales (Gallardo, 2012) o expertos rutinarios en el manejo de las TIC.

\title{
3.- Nuevos roles del docente en las escuelas digitales
}

Ante esta situación los docentes vamos a desempeñar roles más novedosos que los de transmisor de información, como tradicionalmente hemos venido desempeñando. Aunque ello, como señalé en otro momento (Cabero, 2016, p.94) debe ser bien entendido pues

\begin{abstract}
“...no significa que el profesor deje de ser una persona significativa en todo lo referido a la información, por el contrario, y de forma diferente a lo que algunos creen y exponen, las nuevas tecnologías van a llevarlo a que desempeñe nuevas funciones relacionadas con ésta, que irán desde buscar información en la red para adaptarla a las necesidades generales de sus estudiantes o a las necesidades y demandas concretas que a la hora de la evolución del proceso de aprendizaje se vayan presentado. Sin olvidarnos que el profesor desempeñará un fuerte papel en la formación del sujeto para que evalúe y seleccione la información pertinente, de la voluminosa que se le ofrecerán por diversos medios".
\end{abstract}

Uno de los roles más significativos es el de diseñador de TIC y de los entornos de aprendizaje. Ello implicará que el profesor se convierta en un diseñador de situaciones de aprendizaje, que deberán de girar en torno al estudiante y a que este adquiera los conocimientos previstos y por tanto, el aprendizaje. Dicho en otros términos el docente se convertirá en un facilitador del aprendizaje desde la perspectiva en que lo importante no será el entorno en que se produzca, sino que el mismo se encuentre a disposición del estudiante para que este llegue a aprender. El profesor, de esta forma, pasa de ser un experto en contenidos a un facilitador del aprendizaje, lo cual le va a suponer que realice diferentes cuestiones como son: diseñar experiencias de aprendizajes para los estudiantes, ofrecer una estructura inicial para que los alumnos comiencen a interaccionar, animar a los estudiantes hacia el autoestudio o diseñar diferentes perspectivas sobre un mismo tópico.

Ahora bien, también el docente va a jugar un papel importante en el diseño de medios, materiales y recursos adaptados a las características de sus alumnos, materiales que no sólo serán elaborados por él de forma independiente, sino en 
colaboración, tanto con el resto de compañeros involucrados en el proceso, como con otra serie de expertos. Desde esta perspectiva, el profesor deberá aprender a trabajar en equipo y en colaboración con otros profesionales. Cada vez es más usual la formación de consorcios entre diferentes colectivos de profesores para la organización de cursos de forma conjunta, en los cuales cada uno de ellos aporta sus conocimientos más relevantes y todos salen beneficiados por la suma de los esfuerzos realizados.

Otro de los roles a desempeñar por el docente y debido, en cierta medida, a la amplitud de tecnologías existentes, será la de seleccionador de TIC y creador de contenidos, y para ello podrá apoyarse en una serie de aspectos como son:

“• La selección de los medios debe hacerse teniendo en cuenta los objetivos y contenidos que se desean alcanzar y transmitir.

- Las predisposiciones que el alumnado y el profesorado tengan hacia el medio, pueden condicionar los resultados que se obtengan y, en consecuencia, debe de ser uno de los criterios a movilizar para su puesta en acción.

- Contemplar las características de los receptores: edad, nivel sociocultural y educativo, inteligencias múltiples, estilos cognitivos,...

- El contexto instruccional y físico es un elemento condicionador, facilitando o dificultando la inserción del medio.

- Las diferencias cognitivas entre los estudiantes pueden condicionar los resultados a alcanzar y las formas de utilización.

- Los medios deben propiciar la intervención sobre ellos.

- Las características técnicas y sémicas del medio y sus parámetros de cualidades es una dimensión a considerar, aunque no la única y posiblemente la no más significativa.

- En la medida de lo posible seleccionar medios que permitan la participación del profesorado y el alumnado en la construcción de los mensajes.

- Analizar los mensajes contemplando no sólo su capacidad como canal, sino también las características de los mensajes que transmite, y sobre todo contemplando los valores transferidos.

- No marginar socialmente a los estudiantes, por imponer tecnologías a las que no todos tienen posibilidad de acceder.

- Las calidades técnicas, facilidad y versatilidad del medio, deben ser también contempladas.

- Seleccionar medios de fácil utilización.

- En la medida de lo posible seleccionar medios que puedan relacionarse con otros" (Cabero, 2016, p.98).

Los roles que estamos comentando nos llevarán a que el docente del futuro deberá saber trabajar en equipo de forma colaborativa, creo que los tiempos del 
profesor aislado tienen que pasar a la historia, pero para ello también es necesario que se resuelvan los problemas salariales, que hacen que los profesores estén cambiando de un puesto laboral a otro.

\section{4.- Unas reflexiones finales}

Antes de finalizar nos gustaría realizar unas referencias finales que consideramos van a condicionar el papel que el docente desempeñe en las escuelas digitales y que les deben de llevar a reflexionar sobre su papel en las mismas.

Una de ellas se refiere a la "brecha digital", ya que como señala la UNESCO (UNESCO, 2005) forma parte de los cinco obstáculos que impiden el desarrollo de la sociedad del conocimiento: 1) la brecha digital; 2) la brecha cognitiva; 3) la concentración de conocimiento y más concretamente de los conocimientos de vanguardia y de las inversiones importantes en los campos de la ciencia y la educación en determinadas zonas geográficas; 4) en principio, el conocimiento tiene que ser objeto de un aprovechamiento compartido; y 5) el auge de sociedades en las que el conocimiento sea un bien común compartido se ve agravado en la actualidad por una serie de disparidades sociales, nacionales, urbanas y familiares...

Respecto a su conceptualización bien estará adentrarnos en la definición de la brecha digital, y en este aspecto se podría entender por ella la diferenciación producida entre aquellas personas, instituciones, sociedades o países, que pueden acceder a las TIC de forma general y a Internet de manera particular, y aquellas que no pueden hacerlo (Cabero, 2015b); es decir, puede ser definida en términos de la desigualdad de posibilidades que existen para acceder a la información, al conocimiento y la educación mediante las TIC, sea por motivos económicos, de edad, de género, de raza, de ubicación geográfica, u otros motivos.

Ahora bien esta definición se ha ido transformando y en la actualidad podemos hablar de tres tipos de brecha digital:

- La primera podríamos considerarla de carácter instrumentaltecnológica, y es aquella que se produce como consecuencia de no tener acceso a las tecnologías por los motivos anteriormente señalados.

- La segunda, es aquella que se produce en los casos en los cuales existiendo la tecnología las personas no tienen formación para acceder a ellas y utilizarlas.

- $\quad$ Y la tercera, está relacionadas con los diferentes tipos de usos que las personas hacen de las tecnologías, y en este sentido podríamos hablar de usos elementales y usos más significativos y novedosos.

Si las soluciones al primer tipo de brecha son de carácter económicos, político, e institucional, para las otras dos las soluciones tienen que venir desde la educación, mediante la formación de los alumnos en alfabetización digital, que debe implicar:

“- Dominen el manejo técnico de cada tecnología (conocimiento práctico del hardware y del software que emplea cada medio). 
- Posean un conjunto de conocimientos y habilidades específicos que les permitan buscar, seleccionar, analizar, comprender y recrear la enorme cantidad de información a la que se accede a través de las nuevas tecnologías.

- Desarrollen un cúmulo de valores y actitudes hacia la tecnología de modo que no se caiga ni en un posicionamiento tecnofóbico (es decir, que se las rechace sistemáticamente por considerarlas maléficas), ni en una actitud de aceptación acrítica y sumisa de las mismas.

- Y utilicen los medios y tecnologías en su vida cotidiana no sólo como recursos de ocio y consumo, sino también como entornos para expresión y comunicación con otros seres humanos" (Cabero, 2016, p.192).

Alfabetización que requiere contemplar diferentes aspectos tales como (Cabero, 2016, p.194):

“a) Hablar de alfabetización digital requiere hablar de una alfabetización que supera con creces el mero dominio tecnológico e instrumental de las TIC.

b) Supone no sólo la capacidad de recepción de mensajes, sino también la construcción de los mismos.

c) Implica la capacidad de evaluar y seleccionar, de acuerdo a nuestro proyecto formativo y necesidad, la cantidad de información que a través de las nuevas tecnologías nos están llegando.

d) Capacidad para encontrar información, comprenderla y valorarla.

e) Utilizar los medios y las tecnologías en su vida cotidiana no sólo como recursos de ocio y consumo, sino también como entornos para la expresión y la comunicación con otras personas.

f) Supone comprender la alfabetización como actitud de uso para la comunicación"

Y, por otra parte, supone la capacitación del alumno en una serie de aspectos, tales como:

-"Conocer cuando hay una necesidad de información.

- Identificar la necesidad de información.

- Trabajar con diversidad de fuentes y códigos de información.

- Saber dominar la sobrecarga de información.

- Evaluar la información y discriminar la calidad de la fuente de información.

- Organizar la información.

- Usar la información eficientemente para dirigir el problema o la investigación. 
- Saber comunicar la información encontrada a otros" (Cabero y Llorente, 2006, p.14).

Otro de los aspectos que nos gustaría señalar es la transformación que tenemos que hacer de las TIC, ya que como señala Cabero (2014a) las podemos incorporar desde tres posiciones diferentes, que implican, por una parte darle un sentido y aplicación específica a la enseñanza, destacando en unas su visión transmisoras y en otras su posición creadora, y por otra, que orientan la epistemología desde la que debemos llevar a cabo la formación y el perfeccionamiento del profesorado en estos elementos curriculares. Posiciones que podemos denominar como:

- TIC (Tecnologías de la Información y Comunicación).

- TAC (Tecnologías para el Aprendizaje y el Conocimiento).

- TEP(Tecnologías para el Empoderamiento y la Participación).

Desde la perspectiva de las TIC, estos recursos son fundamentalmente percibidos como facilitadores y transmisores de información y recursos educativos para los estudiantes, que pueden ser adaptados a las necesidades y características independientes de los sujetos, pudiendo conseguir con ellos una verdadera formación audiovisual, multimedia e hipertextual. Desde esta posición, los conocimientos que deberemos tener para su utilización se centrarán fundamentalmente en la vertiente tecnológica e instrumental.

Desde la posición de las TAC, implica su utilización como instrumentos facilitadores del aprendizaje y la difusión del conocimiento. Son pues, vistas no tanto como instrumentos de comunicación, sino como herramientas para la realización de actividades para el aprendizaje y el análisis de la realidad circundante por el estudiante. Se trata de dirigir su utilización hacia usos más formativos, tanto para docentes como para discentes, con el objetivo de aprender de manera más significa y excelente.

Desde esta visión, su significación para la educación vendrá de las estrategias y metodologías que se aplicarán sobre ellas para alcanzar los objetivos previstos y crear nuevas escenografías de comunicación para el aprendizaje. Aquí se trataría de que el docente las movilice no para realizar lo mismo que hace sin ellas, es decir, reproducir modelos tradicionales de enseñanza, sino aplicarlas para crear innovaciones educativas y buscar en su aplicación nuevos usos educativos, para que el alumnado las utilice como instrumentos de formación y conocimiento, y no simplemente como herramientas tecnológicas e instrumentales. Desde esta posición, las competencias que deberá tener el profesorado hay que insertarlas en la práctica educativa y crear con ellas escenografías para la formación. No serán meramente tecnológicas, sino más bien metodológicas para saber aplicar sobre ellas diferentes estrategias para alcanzar diferentes objetivos y competencias. Posiblemente, no necesitemos tener tanta formación para utilizarlas, y sí para saber qué pueden hacer los alumnos y alumnas con ellas para adquirir conocimientos. Y ello pasará por hacernos la siguiente pregunta: ¿cómo puedo utilizarlas para cambiar mi práctica docente?

Por último, desde la posición de las TEP, se trataría de percibirlas no como meros recursos educativos, sino también como instrumentos para la participación y la colaboración de docentes y discentes, que además no tienen que estar situados en el mismo espacio y tiempo. Se parte por tanto de la perspectiva de que el aprendizaje no 
solo tiene una dimensión individual, sino también social, ya que la formación implica aprender en comunidad y ser capaz de interactuar y colaborar para construir el conocimiento. Desde aquí, el rol del docente será el de diseñar la escenografía para el aprendizaje, y para ello la tecnología jugará un papel de mediadora en la construcción del conocimiento y la interacción social. Desde esta posición no debemos olvidarnos de que el aprendizaje ya no se produce solo en las instituciones educativas, sino que cada vez es más ubicuo; de aquí que la función de la escuela sea más la de integrar los diferentes aprendizajes que se producen en contextos diferenciados. Ello nos lleva a repensar el rol de la escuela y de las instituciones de formación. Y supone también que él y el discente sean más proactivos, y por tanto, no solo consuman información, sino que también la creen.

En cierta medida podríamos decir que la utilización de las TIC por los docentes deben buscar nuevas perspectivas a las tradicionales de transmitir contenidos y motivar a los estudiantes, y éstas se deben dirigir a su utilización para: comunicar, conocer, participar y empoderar.

Ahora bien, todas estas nuevas perspectivas de incorporación y utilización de las TIC no son posibles, sin que el profesorado se encuentre capacitado para ello, y al respecto Merma (2008) nos señala que el profesor debe tener un amplio espectro de competencias para saber incorporar las TIC en la práctica educativa. En concreto nos indica: competencias cognoscitivas (que se refiere al conocimiento de su disciplina), competencias técnicas (relacionadas con el dominio de las TIC desde un punto de vista técnico-instrumental), competencias de las aplicaciones pedagógicas de las TIC (centradas en el conocimiento de las posibilidades que ofrecen las TIC como herramientas educativas), competencias metodológicas (referidas a la aplicación de procedimientos adecuados), actitud positiva y crítica, facilitador del proceso de enseñanza-aprendizaje, habilidad para que sus alumnos incorporen las TIC en su aprendizaje, habilidad para elegir adecuadamente los recursos tecnológicos, formarse y capacitarse permanentemente en el uso de las TIC, habilidad para trabajar cooperativamente en redes, y ser un evaluador constante.

Por otra parte, Mishra y Koehler (2006) han propuesto un modelo de análisis del funcionamiento de las TIC en los procesos de enseñanza-aprendizaje que denominan Technological Pedagogical Content Knowledge o TPCK que podríamos denominar en español Conocimiento Tecnológico Pedagógico Disciplinar o CTPD (figura 1).

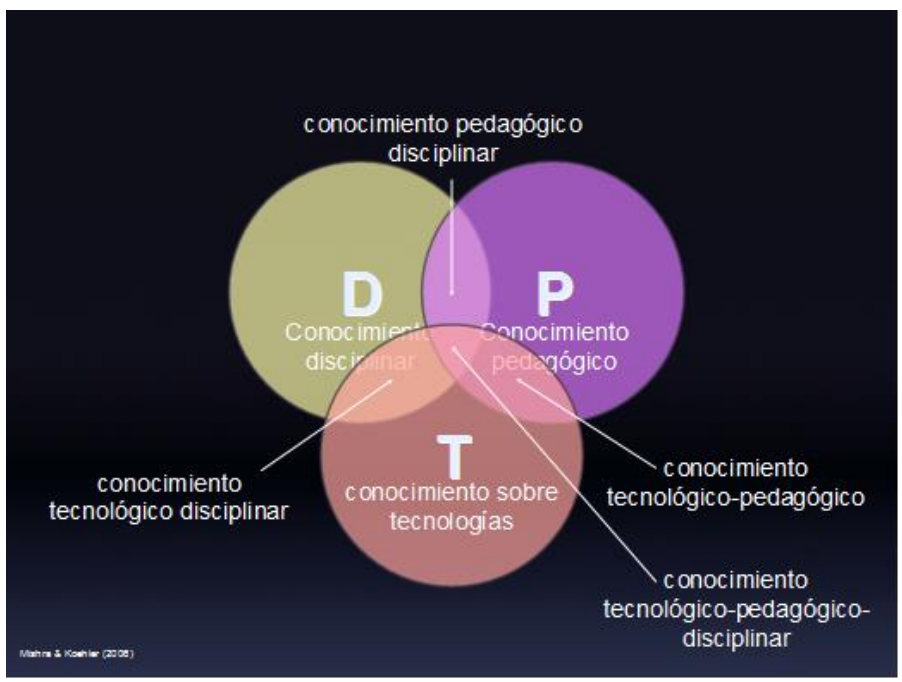

Figura 1. Modelo de Mishra y Koehler (2006). 
Modelo que nos permite señalar la necesidad de contemplar tres grandes dimensiones en los conocimientos que debe poseer el profesor: disciplinar, pedagógico y tecnológico; los cuales no sólo podemos observarlos de forma aislada, sino también en interacción de los tres componentes:

- CC: Conocimiento sobre el contenido de la materia. Claramente los profesores deben conocer los contenidos que deben enseñar, los hechos, los conceptos, sus teorías,...

- $\quad$ CP: Debe conocer los procesos y prácticas del método de enseñanza y cómo se relacionan con el pensamiento y los propósitos educativos.

- CCP: Conocimiento Pedagógico del Contenido: los profesores deben saber cómo enseñar sus materias. Conocimiento pedagógico aplicado a una asignatura.

- $\quad$ CT: Conocimiento de las TIC estándar que se utilizan en la enseñanza.

- $\quad$ CPT: Conocimiento de la utilización de las TIC en los posesos de enseñanza. Cómo la enseñanza, por ejemplo puede cambiar utilizando las TIC y utilizándolas de una forma específica. Hay muchos conocimientos que son fundamentales para la enseñanza, como por ejemplo, cómo aprende y piensa y el conocimiento del dominio de la materia.

CTPC: Conocimiento Tecnológico, pedagógico y de contenido. La enseñanza requiere la comprensión de la representación de conceptos usando la tecnología, técnicas que usan la tecnología desde una perspectiva constructivista para enseñanza el contenido y los conceptos.

Este modelo ha sido validado por Cabero (2014b), Cabero, Marín y Castaño (2015) y Cabero y Barroso (2016), denostando su viabilidad en contextos latinos.

Para finalizar señalar dos aspectos: 1) En definitiva la sociedad de la información requerirá de un nuevo tipo de profesores más adaptados a las necesidades que se le plantearán a la escuela de la sociedad. Y en este sentido o el profesor se sube a la historia o formará parte de ella; y 2) Nos vamos a mover en un terreno de doble velocidad; mientras los cambios tecnológicos son rápidos, dinámicos e incluso fugaces; los cambios educativos y culturales son lentos y apaciguados.

\section{Referencias}

Badia, A., Meneses, J., \& García, C. (2015). Technology use for Teaching and learning. Pixel-Bit. Revista de Medios y Educación, 46, 9-24. doi: 10.12795/pixelbit.2015.i46.01.

Cabero, J. (2014a). Reflexiones sobre la brecha digital y la educación: siguiendo el debate. Inmanecencia, 4(2), 14-26. 
Cabero, J. (2015a). Reflexiones educativas sobre las tecnologías de la información y comunicación (TIC). Tecnología, Ciencia y Educación, 1, 19-27.

Cabero, J. (2015b). Inclusión digital - inclusión educativa. Sinergia, 2, 15-18. Recuperado de http://telebachilleratoenchiapas.gob.mx/wpcontent/uploads/2015/08/SINERGIA 2Edición.pdf

Cabero, J. (2016). Tendencias educativas para el siglo XXI. Madrid: Ediciones CEF.

Cabero, J. (dir.) (2014b). La formación del profesorado en TIC: modelo TPACK. Grupo de Investigación Didáctica: Sevilla.

Cabero, J., \& Barroso, J. (2016). ICT teacher training: a view of the TPACK model / Formación del profesorado en TIC: una visión del modelo TPACK. Cultura y Educación, 28(3). 633-663. doi:10.1080/11356405.2016.1203526.

Cabero, J., \& Barroso, J. (coords.) (2013). La escuela en la sociedad de la información. La escuela 2.0. En J. Barroso \& J. Cabero (coords.), Nuevos escenarios digitales. Las tecnologías de la información y la comunicación aplicadas a la formación y el desarrollo curricular (pp. 21-36). Madrid: Pirámide.

Cabero, J., \& Llorente, M.C. (2006). La rosa de los vientos, Dominios tecnológicos de las TICs por los estudiantes. Sevilla: Grupo de Investigación Didáctica.

Cabero, J., \& Llorente, M.C. (2015). Tecnologías de la Información y la Comunicación (TIC): escenarios formativos y teorías del aprendizaje. Revista Lasallista de Investigación, 12(2), 186-193.

Cabero, J., Marín, V., \& Castaño, C. (2015). Validación de un instrumento de diagnóstico del modelo TPACK (conocimientos tecnológicos, pedagógicos y de contenidos) para la formación del profesorado en TIC. @ tic, 14, 13-22. doi: 10.7203/attic.14.4001

Castaño, J., Duart, J., \& Sancho, T. (2012). Una segunda brecha digital entre el alumnado universitario. Cultura y Educación, 24(3), 363-377.

Durall, E., Gros, B., Maina,M.F. Johnson, L., \& Adams, S. (2012). Perspectivas tecnológicas: educación superior en Iberoamérica 2012-2017. Austin, Texas: The New Media Consortium.

Flores, O., \& Del Arco, I. (2013). Nativos digitales, inmigrantes digitales: rompiendo mitos. Un estudio sobre el dominio de las TIC y estudiantado de la Universidad de Lleida. Bordón, 65(2), 59-74.

Gallardo, E. (2012). Hablemos de estudiantes digitales y no de nativos digitales. Revista de Ciénces de l'educació, XXXVII, 7-21. 
Instituto Tecnológico de Monterrey (2015). Reporte EduTrends. Radar de Innovación Educativa 2015. Monterrey: Tecnológico de Monterrey.

Jenkins, H. (2007). Convergence Cultura. La cultura de la convergencia de los medios de comunicación. Barcelona: Paidós.

Johnson, L., Adams, S., Cummins, M., Estrada, V., Freeman, A., \& Hall, C. (2016). NMC Horizon Report: 2016 Higher Education Edition. Austin, Texas: The New Media Consortium.

Merma, G. (2008). Competencias del profesorado para el uso de las tecnologías de la información y la comunicación en la enseñanza, en el marco del Espacio Europeo de Educación Superior. En R. Roig (dir), Investigación e innovación en el conocimiento educativo actual (pp.317-326). Marfil: Alcoy.

Mishra, P., \& Koehler, M. (2006). Technological Pedagogical Content Knowledge: A Framework for Teacher Knowledge. Teachers College Record, 108, 10171054.

Pons, B. (2013). Disseny d'un programa de capacitació en competëncies bäsiques TIC per alumnes de secundäria (Tesis doctoral inédita). Palma de Mallorca: Universitat de les Illes Balears.

Prensky, M. (2011). Enseñar a nativos digitales. Madrid: SM.

Romero, M., \& Minelli, J. (2011). La generación net se tambalea. Percepción del dominio de las TIC de estudiantes de magisterio. Revista Teoría de la Educación: Educación y Cultura en la Sociedad de la Información, 12(3), 265-283.

Ryberg, T., Dirckink-Holmfeld, L., \& Jones, C. (2011). Catering to the needs of the "digital natives" of educating the "net generation"? In J. L. Mark \& C. McLoughlin, Web 2.0. Based e-learning applijing social informatics for teatiary teaching (pp. 301-318). New York: Information Science Reference.

Thompson, P. (2013). The digital natives as learners: technology use patterns and approaches to learning. Computers \& Education, 65, 12-33. doi: 10.1016/j.compedu.2012.12.022

UNESCO (2005). Hacia las sociedades del conocimiento. París: UNESCO. 